\title{
Supervaluationism and the timeless solution to the foreknowledge problem
}

\author{
PABLO COBREROS \\ Department of Philosophy University of Navarra \\ pcobreros@unav.es
}

\begin{abstract}
If God knew I were going to write this paper, was I able to refrain from writing it this morning? One possible response to this question is that God's knowledge does not take place in time and therefore He does not properly fore-know. According to this response, God knows absolutely everything, it's just that He knows everything outside of time. The so-called timeless solution was one of the influential responses to the foreknowledge problem in classical Christian Theology. This solution, however, seemed to lose support in the recent debate. For example, Pike claims that "the doctrine of God's timelessness entered Christian Theology (only) because Platonic thought was stylish at the time" (Pike, 1970,190) and Hasker (2001) catalogues this as one of the minor solutions to the problem. One possible source for this general attitude towards timelessness is the thought that the very idea of timelessness is incoherent. In this paper I argue that that the timeless solution to the foreknowledge problem is congenial with the supervaluationist theory of branching time and that this formal framework provides, in fact, a precise characterization of the timeless solution to the foreknowledge problem. The views presented in this paper are in line with those of Kretzmann and Stump (1981), Leftow (1991) and De Florio and Frigerio (2015).
\end{abstract}

Keywords: divine foreknowledge; omniscience; timelessness; indeterminist time; supervaluationism. 


\section{The foreknowledge problem}

Suppose Jones mowed his lawn last Saturday and God foreknew that. ${ }^{1}$ Then, at some point before Saturday, say on Thursday, God believes that Jones will mow his lawn on Saturday. Then Jones' ability to refrain from mowing his lawn before Saturday, say on Friday, is either (a) the ability of making God having a false belief or (b) the ability to influence on someone's past beliefs or (c) the ability to turn into non-existence someone who existed in the past. Neither of (a) to (c) describes a real ability of Jones'. Therefore either Jones does not have the ability to refrain from mowing his lawn on Saturday or God does not foreknow that Jones will mow his lawn on Saturday (see Pike (1965)). Consider the following abbreviations:

$t_{n}: A \quad \rightarrow \quad A$ holds in time $t_{n}$

$[\mathrm{G}] A \quad \rightarrow \quad$ God knows $A$

$\square A \quad \rightarrow \quad$ It is necessary that $A$

$\square \mathrm{A} \quad \rightarrow \quad$ It is accidentally necessary that $A$

Then the argument can be made more explicit in the following way, ${ }^{2}$
1. $t_{0}:[\mathrm{G}]\left(t_{2}: J\right)$
[Assumption]
2. $\square\left(t_{0}:[\mathrm{G}]\left(t_{2}: J\right)\right)$
[From 1 by NP]
3. $\square\left(t_{0}:[\mathrm{G}]\left(t_{2}: J\right) \supset t_{2}: J\right)$
[Instance of EO]
4. $\square\left(t_{2}: J\right)$
[From 2,3 by TN]

1 I would like to express my gratitude to the Classical Theism Project https://classicaltheismproject.wordpress.com/ for a summer stipend in 2015. This paper also benefited from the discussion in the Cluster Group on Analytic Theology http://www.unav.edu/en/web/ facultad-de-filosofia-y-letras/analytic-theology held in the University of Navarra in 2015. Thanks finally to the project Non-Transitive Logics: A New Approach to Paradoxes, funded by the MINECO (Government of Spain) Grant $n^{\circ}$ : FFI2013-46451-P.

2 This argument corresponds to steps (1) to (6) of the argument in (Zagzebski 2011, sec. 1). The argument seems to go back to, at least, the American philosopher Jonathan Edwards (Prior 1967, 113-4). 
The inferences appealed to in the argument are the following,

NP $t_{n}: A \vdash \square\left(t_{n}: A\right) \quad$ (for any $\mathrm{n}<\mathrm{p}$ where $t_{p}$ is present time)

EO $\vdash \square\left(t_{n}:[\mathrm{G}] A \supset A\right) \quad$ (for any $\mathrm{n}$ )

$\mathrm{TN} \square A, \square(A \supset B) \vdash \square B$

NP expresses the "necessity of the past": that anything that already occurred is now accidentally necessary: it's not necessary that Caesar crossed the Rubicon but once he did, there's nothing we can do today to change that it happened. EO records the (tensed) essential omniscience of God: if God believes $A$ (at any time $n$ ) then $A$. TN is the principle of transfer of necessity according to which if $A$ is accidentally necessary and necessarily if $A$ then $B$, it follows that $B$ is also accidentally necessary.

The explanation of the argument is as follows. Suppose that we are talking in time $t_{1}$, after time $t_{0}$ but prior to time $t_{2}$. Step 1 states that God knows in time $t_{0}$ that in time $t_{2}$ Jones mows his lawn. Since $t_{0}$ is already past, it is now accidentally necessary that God knows in time $t_{0}$ that in time $t_{2}$ Jones mows his lawn. Step 3 is an instance of EO. From that conditional in step 3 and the accidental necessity of its antecedent (step 2) it follows that it is today accidentally necessary that in $t_{2}$ Jones mows his lawn (but if it is today accidentally necessary, then there's nothing Jones, or even anyone of us, to change the fact that Jones mows his lawn in $t_{2}$ ).

In this paper we will use a propositional language with simple tenses ' $\langle+\rangle$ ' and ' $[+]$ ' informally meaning 'at some time in the future' and 'at every time in the future' respectively (' $\langle-\rangle$ ' and '[-]' will be used to talk about the past.) Although with this language we don't have explicit reference to times, unlike the language in the argument above, we might still capture its essence in the simple-tense language. The semantics we will give to $\square$ is simple: $\square A$ is true in time $t$ just in case $A$ is true everywhere ${ }^{3}$ the reasons is that $\square$ is supposed to be a form of analytic necessity (see (Pike 1965, 35)). The fundamental idea is the following interpretation of $\square$ : $\square A=\mathrm{df}[+]\langle-\rangle A$

3 More specifically, given an interpretation $\langle\mathcal{T},<, \llbracket \cdot \rrbracket\rangle, \square A$ is true in time $t \in \mathcal{T}$ just in case $A$ is true in every $t \in \mathcal{T}$; see section 2 for the semantics. 
Since $\square$ means something like: it does not matter how the world evolves, it won't be the case that it didn't happen. Now this interpretation is not fully faithful to the idea of the necessity of the past in a, I want to argue, harmless way. The idea of the necessity of the past involves reference to a particular time: if $A$ took place at time $t$ in the past, then it will always be the case that $A$ took place at time $t$. Under the definition above, if $A$ is accidentally necessary, that guarantees that it will always be true that $A$ was true but not necessarily at the same past time. This difference rests crucially in an expressive limitation of our simple modal language; the difference, however, is harmless in the sense that we can add some information to reach a similar effect to that of naming times. ${ }^{4}$ Suppose for example that I want to express that it is accidentally necessary that Jones mows his lawn (due to the fact that Jones is now doing that). Then I can write:

\section{$\square J \wedge \neg\langle-\rangle J$}

In this case, if $\square J$ is true, that is due to something that is happening today. We can see this strategy as a way to force $J$ to make reference to the relevant fact (a fact that is happening today) without explicit naming of times. Given the above qualifications, the final shape of the argument is the following,

0. $\neg\langle-\rangle J \wedge \neg \mathrm{J}$

1. $[\mathrm{G}]\langle+\rangle J$

[Assumption]

2. $\square([G]\langle+\rangle)$ [From 1 by NP]

3. $\square([\mathrm{G}]\langle+\rangle J \supset\langle+\rangle) \quad$ [Instance of EO]

4. $\langle+\rangle J$ [From 2, 3 by TN]

5. $\mathbb{U}\langle+\rangle J$ [From 0 and 4]

We added premise 0 for the reasons just given; in that way, we guarantee that if $\langle+\rangle J$ is accidentally necessary, that is due to something that is hap-

4 Modal logics with explicit reference to times are known as hybrid logics. We avoid a hybrid language in order to keep technicalities to a minimum. 
pening in the immediate future (and not, for example, because J already occurred in the past). For the final line, ' $\mathbb{U} A$ ' means ' $A$ is unavoidable'. Steps 1 to 4 are based on a rewriting of the principles above according to our previous remark about $\square$,

$$
\begin{aligned}
& \mathrm{NP} A \vdash[+]\langle-\rangle A \\
& \mathrm{EO} \vdash \square([\mathrm{G}] A \supset A) \\
& \mathrm{TN}[+]\langle-\rangle A, \square(A \supset B) \vdash[+]\langle-\rangle B
\end{aligned}
$$

\section{Branching time and supervaluationism}

In this section we present Prior-Thomason semantics for indeterminist time (in Thomason (1970)) for the simple modal language of the last argument. ${ }^{5}$ An interpretation for this language is a structure $\langle\mathcal{T},<, \llbracket \cdot \rrbracket\rangle$ where,

$\mathcal{T} \neq \varnothing$

$<$ is a tree order on $\mathcal{T}$

$\llbracket \cdot \rrbracket$ is a function: $\operatorname{Var} \times \mathcal{T} \rightarrow\{1,0\}$

A (strict) partial order $<$ on $\mathcal{T}$ is a relation between the elements of $\mathcal{T}$ that is irreflexive and transitive. Such an order is a tree-order on $\mathcal{T}$ if, in addition:

for any $t, t^{*}$ and $t^{* *}$ if $t^{*}<t$ and $t^{* *}<t$ then either $t^{*}<t^{* * *}$ or $t^{* *}<t^{*}$.

This last condition amounts to the idea that a tree-order is a partial order that is "linear to the left". This idea, in turn, seems to capture the intuitive

5 I discovered, after writing much of this paper, that De Florio and Frigerio (2015) also make use of the formal framework of Thomason to discuss the question of foreknowledge. Although we agree in the philosophical background, the discussions are quite different in, at least, two respects. We both add further structure to Thomason's semantics, but they add a dynamic view of models that interact with a "NOW" operator whereas I intend just to see how to define a truth-predicate coherent with Thomason's semantics. Second, the scope of the paper is different for, whereas theirs discuss a broader range of topics, this paper restricts the attention to the formalization of the foreknowledge problem. 
asymmetry between past and future: for any $t \in \mathcal{T}$ there is a set $\mathcal{H}_{t}$ of histories containing $t$; these histories agree up to $t$ and possibly disagree after $t$. (A history is a subset $\mathcal{T}^{*} \subseteq \mathcal{T}$ that is linearly ordered by <; this is sometimes called a maximal chain.) The tree-order, therefore, seems to rule out Ockhamist solutions. ${ }^{6}$

Figure 1. Tree-like structure

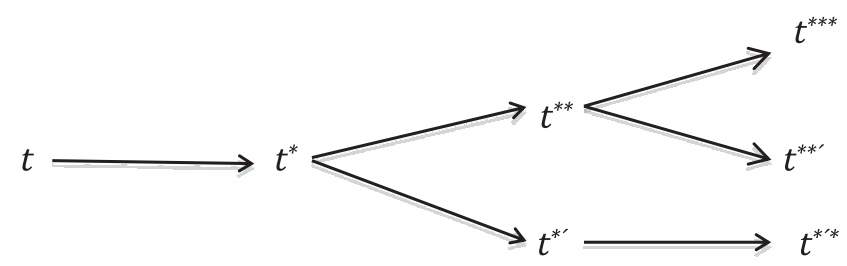

The function $\llbracket \cdot \rrbracket$ is a bivalent assignment of truth-values to propositional variables relative to each $t \in \mathcal{T}$. Given that the same time $t$ might belong to multiple histories, the truth-conditions for a formula $A$ will be relative, not just to a given time $t$ but also to a given history $h$ such that $t \in h$ :

$\llbracket \neg A \rrbracket_{\mathrm{t}}^{\mathrm{h}}=1$ just in case $\llbracket A \rrbracket_{\mathrm{t}}^{\mathrm{h}}=0$

$\llbracket A \wedge B \rrbracket_{\mathrm{t}}^{\mathrm{h}}=1$ just in case $\llbracket A \rrbracket_{\mathrm{t}}^{\mathrm{h}}=\llbracket B \rrbracket_{\mathrm{t}}^{\mathrm{h}}=1$

$\llbracket\langle-\rangle A \rrbracket_{\mathrm{t}}^{\mathrm{h}}=1$ just in case $\exists \mathrm{t}^{*} \in \mathrm{h}$ such that $\mathrm{t}^{*}<\mathrm{t}$ and $\llbracket A \rrbracket_{\mathrm{t}^{*}}^{\mathrm{h}}=1$

$\llbracket\langle+\rangle A \rrbracket_{\mathrm{t}}^{\mathrm{h}}=1$ just in case $\exists \mathrm{t}^{*} \in \mathrm{h}$ such that $\mathrm{t}<\mathrm{t}^{*}$ and $\llbracket A \rrbracket_{\mathrm{t}^{*}}^{\mathrm{h}}=1$

This semantics works fine for either classical or ' $\langle-\rangle$ ' operators but there is an issue with ' $\langle+\rangle$ ' formulas. Given that a tree-order might be "non-linear to the right”, a formula ' $\langle+\rangle A$ ' might receive different truth-values relative to different histories (see figure 2 ). Now if ' $\langle+\rangle A^{\prime}$ ' is true in $t$ relative to history $h[t \in h]$ and false relative to $h^{*}\left[t \in h^{*}\right]$, what is the final truth-value of

6 See Saunders (1966), Adams (1967) and a number of replies in that journal. The choice of a tree-order comes with the underlying assumption that future indeterminacy should be accounted for in terms of "branching time". 
' $\langle+\rangle A$ ' in $t$ ? Thomason's strategy (Thomason 1970, 272) is considering that, in such a case, the formula is neither true nor false. ${ }^{7}$

Figure 2. Open Future

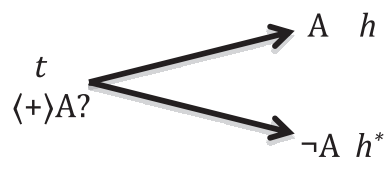

A supervaluation is a partial valuation based on a set of complete valuations. A time $t$ determines a set $\mathcal{H}_{t}$ of histories (the set of histories that pass over $t$ ). Each history $\mathrm{h} \in \mathcal{H}_{t}$, in turn, provides a complete valuation for formulas of the language (including ' $\langle+\rangle$ ' formulas) relative to time $t$. For this reason, the tree-like structure of time is a natural context to define a supervaluation:

$A$ is supertrue at time $t$ just in case for all $h \in \mathcal{H}_{t}, \llbracket A \rrbracket_{\mathrm{t}}^{\mathrm{h}}=1$.

In general, logical consequence is a matter of necessary preservation of truth. Since our relevant notion of truth is that of supertruth, logical consequence is defined accordingly (the subscript PT is for Prior-Thomason tense logic):

$\Gamma \vDash_{\mathrm{PT}} A$ just in case, there is no interpretation $\langle\mathcal{T},<, \llbracket \cdot \rrbracket\rangle$ with $t \in \mathcal{T}$ such that: $\forall \mathrm{h} \in \mathcal{H}_{\mathrm{t}} \forall \mathrm{B} \in \Gamma, \llbracket \mathrm{B} \rrbracket_{\mathrm{t}}^{\mathrm{h}}=1 \& \exists \mathrm{h} \in \mathcal{H}_{\mathrm{t}} \llbracket \mathrm{A} \rrbracket_{\mathrm{t}}^{\mathrm{h}}=0$

In words, an argument is valid just in case there is no interpretation and time $t$ such that all premises are supertrue and the conclusion is not.

7 Alternatively, we could consider that it is both true and false. Subvaluationism, the dual theory of supervaluationism, has precisely this effect. 


\section{Retrospective determinacy and a supervaluationist truth-predicate}

A characteristic feature of supervaluationism generally speaking is that it makes compatible classical logic with truth-value gaps. ${ }^{8}$ In the case of Prior-Thomason's temporal logic supervaluationism comes with some additional validities, ${ }^{9}$ one of which is the following inference:

$$
A \vDash_{\mathrm{PT}}[-]\langle+\rangle A
$$

For suppose $A$ is true in actual time $t$. Then $\langle+\rangle A$ is true in any time $t^{*}<t$ of any history $h \in \mathcal{H} t$; that is, $\langle+\rangle A$ is supertrue at any such time $t$ (relative to the set of histories $\mathcal{H}_{t}$ ).

The validity of this inference is connected with the so-called problem of retrospective determinacy (see Loss (2012)). According to the previous semantics, the utterance of a statement $\langle+\rangle A$ might be neither true nor false at some time $t$. If, however, according to some later time $t^{*} A$ comes out true, we can "looking backwards" consistently say that the utterance of $\langle+\rangle$ $A$ was true in $t$ (this last is recorded in the validity of the inference above). Explaining how can this inference be valid in the presence of truth-value gaps involves a peculiar form of relativism: the same proposition (not just the same sentence) might be both neither true nor false and true at the same time relative to different circumstances of evaluation. ${ }^{10}$

Thomason takes this inference to show that the notion of supertruth is different from that of unavoidability. Thomason considers the following definition of unavoidability,

$\llbracket \mathbb{U A} \rrbracket_{\mathrm{t}}^{\mathrm{h}}=1$ just in case $\forall \mathrm{h}^{*} \in \mathcal{H}_{\mathrm{t}} \llbracket \mathbb{U} A \rrbracket_{\mathrm{t}}^{\mathrm{h}^{*}}=1$

8 In addition to Thomason's paper van Fraassen (1966) and Fine (1975) are classical examples of the application of supervaluationism in different contexts. See Keefe (2000) for a more contemporary defence of supervaluationism. See Cobreros and Tranchini (2014) for recent discussion on this feature of supervaluationism.

$9 \quad \vDash \mathrm{PT}\langle+\rangle \mathrm{A} \vee\langle+\rangle \neg \mathrm{A}$ is a particularly nice example.

10 This point is convincingly vindicated by Loss (2012) in response to an objection of MacFarlane (2008). 
And wonders whether this is not in fact an object language expression of the idea of supertruth (Thomason 1970, 278). As Thomason points out, under this definition truth seems to collapse with unavoidability since the definition of $\mathbb{U}$ seems to mirror in the object language the definition above of supertruth. Furthermore, the following pair of inferences are valid:

- $\mathbb{U A} \vDash \mathrm{A}$

- $\mathrm{A} \vDash_{\mathrm{PT}} \mathbb{U A}^{11}$

Thomason, however, argues that, despite initial appearances, truth and unavoidability (or inevitability, as he says) are different. He seeks to show this difference defining a new operator $\mathbb{T}$ for truth:

$\llbracket \mathbb{T A} \rrbracket_{t}^{\mathrm{h}}=1$ just in case $\llbracket A \rrbracket_{t}^{\mathrm{h}}=1$ (Thomason 1970, 278)

This is a transparent truth-predicate, allowing for full substitutivity between $A$ and ' $T A$ '. This means, among other things, that the inference,

$A \vDash_{\text {PT }}[-]\langle+\rangle A$

remains valid. The same, however, cannot be said of unavoidability since, $A \not[-]\langle+\rangle A$.

With this different logical behaviour, Thomason nicely puts the subtle difference between truth and unavoidability:

Our theory thus allows (indeed forces) us to say that having been true is different from having been inevitable, as far as future-tensed statements go. The latter is not a consequence of the former,

$$
[-] \mathbb{T}\langle+\rangle A \not \#_{\mathrm{PT}}[-]\langle+\rangle A,
$$

11 The inferences don't entail the triviality of $\mathbb{U}$ because of a failure of the deduction theorem; in particular, $A \supset \mathbb{U} A$. 
because in an assertion that it was true that a thing would come about, truth is relative to events up to the present, whereas in an assertion that it was inevitable that a thing would come about, inevitability is judged relative to some time in the past. (p. 279)

I think Thomason's explanation about the difference between truth and unavoidability hits the nail on the head. I don't think, however, Thomason's definition of ' $\mathbb{T}$ ' reflects the notion of truth working in the semantics, since supertruth allows for truth-value gaps but with the above definition we have, among other things, $\vDash_{\mathrm{PT}} \mathbb{T} A \vee \neg \mathbb{T} A$.

Thomason's explanation of the difference between truth and unavoidability contains the key to provide an object-language characterization of supertruth. The driving idea is the following. The evaluation, in a given time, of a sentence containing tense operators requires moving forwards or backwards along the time structure. If times are linearly ordered, there is a single relevant history and the "movement" in search of times for evaluation reduces always to that history. If time has a tree-like structure, the point at which we start the evaluation, what we might call the perspective, determines the histories relevant for the evaluation of the sentence. The sentence "Jones will mow his lawn" is neither true nor false on Friday, when Friday is the starting point of evaluation (when Friday is the perspective). The sentence "Jones will mow his lawn" is true on Friday, when Saturday is the perspective.

The idea can be expressed in a more formal style saying that, in treelike structures, the evaluation of tensed sentences involves a double reference to time. ${ }^{12}$ One of the reference times is the time at which we evaluate a given sentence (what we will call the evaluation time), the second reference time is the perspective.

In the previous semantics, we made explicit reference to the evaluation time, but we didn't do the same with the perspective. The reason, I think, is

12 This idea appears also in Loss (2012). The double-time-reference is explicitly endorsed and defended in Belnap and Green (1994) and Perloff and Belnap (2011). These authors, however, reject the supervaluationist treatment of future contingent statements. 
that we tend to take for granted that the perspective, the time at which the evaluation process begins, is identical with the time at which the sentence under evaluation is uttered, the time of assertion (see Perloff and Belnap (2011)). This makes perfect sense. As temporal beings, our actions, and particularly speech acts like assertions, take place in a particular time and are therefore connected to a particular perspective. It is not unthinkable, however, that perspective and time of assertion be different, as we shall point out later.

Given the previous remarks, we redefine the truth predicate as relative to a perspective and also redefine unavoidability accordingly.

$\llbracket \mathbb{T A} \rrbracket_{\mathfrak{t}_{[p]}^{\mathrm{h}}}^{\mathrm{h}}=1$ just in case case $\forall \mathrm{h}^{*} \in \mathcal{H}_{\mathrm{p}} \llbracket \mathrm{A} \rrbracket_{\mathrm{t}[\mathrm{p}]}^{\mathrm{h}^{* \prime}}=1$

$\llbracket \mathbb{U A A} \rrbracket_{t[p]}^{\mathrm{h}}=1$ just in case case $\forall \mathrm{h}^{*} \in \mathcal{H}_{\mathrm{t}} \llbracket \mathrm{A} \rrbracket_{\mathrm{t}[\mathrm{t}]}^{\mathrm{h}^{*}}=1$

The intended meaning of this truth operator is the following: a sentence $A$ is true, relative to a given perspective $p$, just in case $A$ is "settled" according to that perspective (true for all histories that pass over the perspective). Following our example, the statement 'It is true that Jones will mow his lawn' is false from Friday's perspective but true from Saturday's perspective. The definition of unavoidability intends to register the fact that, unlike truth, this notion is relative to the time of evaluation (as Thomason points out 'inevitability is judged relative to some time in the past.') and hence the predicate takes into account all histories from the time of evaluation on. $\mathbb{T}$ and $\mathbb{U}$ are certainly similar in that both are defined relative to a set of histories, but might differ in exactly what set. That set is guaranteed to be the same when time of evaluation and perspective coincide but not otherwise.

We end up this section with a small remark about logical consequence and its relation to time. Logical consequence can be characterized as constraining what we are in a position to deny given what we assert. If, for example, I assert $A \wedge B$, I'm not in a position to deny $A$. In the context of tensed sentences and, furthermore, assuming that truth is relative to a perspective, we should make explicit the role of time. Logical consequence 
constraints what we are in a position to deny in time $t$ given what we assert in $t$. If, for example, I presently assert $A \wedge B$, I'm not presently in a position to deny $A$. For this reason we set the same time for the initial time of evaluation and perspective in the definition of logical consequence:

$\Gamma \vDash A$ just in case, there is no interpretation $\langle\mathcal{T},<, \llbracket \cdot \rrbracket\rangle$ with $t \in \mathcal{T}$ such that: $\forall \mathrm{h} \in \mathcal{H}_{\mathrm{t}} \forall \mathrm{B} \in \Gamma, \llbracket \mathrm{B} \rrbracket_{\mathrm{t}[\mathrm{t}]}^{\mathrm{h}}=1 \& \exists \mathrm{h} \in \mathcal{H}_{\mathrm{t}} \llbracket \mathrm{A} \rrbracket_{\mathrm{t}[\mathrm{t}]}^{\mathrm{h}}=0$

Given the previous definitions, here are some interestingly valid and invalid inferences involving $\mathbb{T}$ and $\mathbb{U}$ :

- $U A \vDash \mathbb{T} A \& \mathbb{T A} \vDash \mathbb{U A}$

- $A \vDash[-] \mathbb{T}\langle+\rangle \mathrm{A}$

- $A \not[-] U\langle+\rangle A$

- $\mathrm{A} \not[-] \mathbb{U T}\langle+\rangle \mathrm{A}$

\section{The timeless solution to the foreknowledge problem}

According to some classical authors like Anselm, Boethius and Aquinas, God's eternality should be understood as God's existing "outside time" so that we cannot attribute temporal properties to God. In this sense, the problem of foreknowledge seems to disappear: it cannot be properly said that God foreknows anything. So in the argument above, the assumption in line 1, that God knows that Jones will mow his lawn, seems illegitimate. Things, however, are not so easy. Since for these authors, like for many theistic positions, God communicates with creatures and from time to time makes infallible predictions (prophecies). Furthermore, in the classical view, God's omniscience is not restricted by the openness of the future, rather, God knows all things, past, present and future, in a simultaneous present (Kretzmann and Stump 1981, 434). So God knows whether Jones will mow his lawn and the question raises again, how can the timeless view address the argument? 
Note that given the supervaluationist semantics for branching time, the argument is valid. The principle of necessity of the past is valid, so that proposition (1): 'God knows $J$ ', entails proposition (2): 'it will always be true that God knew I'. Our interpretation of as $\square$ true everywhere is strong enough to guarantee the validity of transfer of necessity, since the truth of $[+]\langle-\rangle A$ and the falsity of $[+]\langle-\rangle B$ at the same time $t$ require some time $t^{*}$ where $A$ is true and $B$ false, contrary to the truth of $\square(A \supset B) .{ }^{13}$ The final step, from 0 and 4 to 5 is, once again, valid. ${ }^{14}$ Informally, if $J$ is not true today, nor in any past time (assumption $0: \neg\langle-\rangle J \wedge \neg J$ ), then that $[+]\langle-\rangle\langle+\rangle J$ is supertrue means that all future histories contain one immediately succeeding time where $J$, in which case $\mathbb{U}\langle+\rangle J$, it is unavoidable that Jones will mow his lawn.

In our definition of logical consequence above we assumed that the perspective coincides with the time of assertion, but what happens when this is not the case? Consider the case of a time traveller. A crucial incident in the plot of Back to the Future II is the travel to the past of the Sports Almanac containing all the results in a number of competitions from 1950 to 2000. When old Tannen gives the Sports Almanac to young Tannen in 1954, he asserts 'UCLA will win 19-17'. Is this assertion true? Well, it's true from old Tannen perspective but at this point the perspective and the time of assertion are different. When this happens, truth might fail to be factive in the sense that the following inference is invalid,

$$
\mathbb{T A} \vDash / \mathrm{A}
$$

13 Here is a more detailed explanation. Suppose there is some time $t$ at which $[+]\langle-\rangle A$ and that $\square(A \supset B)$ are supertrue but $[+]\langle-\rangle B$ is superfalse. According to the last, for any history $h \in \mathcal{H}_{t}$ there is some time $t<t^{*}$ such that $\langle-\rangle B$ is false in $t^{*}$; this last in turn means that $B$ is false at all times prior to $t^{*}$. Call $h^{\prime \prime}$ to any such history. Since $[+]\langle-\rangle A$ is supertrue at $t$, it is true, in particular, relative to history $h^{\prime \prime}$. Therefore, at time $t^{* \prime}\langle-\rangle A$ is true and this requires a time $t^{\prime \prime \prime}<t^{\prime \prime}$ such that $A$ is true in $t^{* * *}$. But by what we said above $\mathrm{B}$ is false at $t^{* * * *}$ and, therefore, the conditional $A \supset B$ is also false at $t^{m * n}$, contrary to the assumption that $\square(A \supset B)$.

14 The validity of this step rests on Thomason's supervaluationist version of Prior's logic; that inference might not be valid without the assumption that the property preserved by logical consequence is supertruth. 
Under old Tannen's perspective, it is true that UCLA will win 19-17, but this does not entail that it is a fact (that it is true taking that point in 1954 as the perspective).

According to the timeless solution, God does not share our perspective of time. My thesis is, therefore, that the timeless solution to the foreknowledge problem should be understood along these lines: when God asserts something (or better: when we claim God is in a position to assert something), the perspective and the time of assertion do not coincide. In this situation, the truth predicate above is no longer factive. Taking the argument once again, that God knows that Jones will mow his lawn entails that it is true that Jones will mow his lawn, but this, in turn, does not entail that Jones will mow his lawn.

\section{References}

Adams, Marylin M. 1967. “Is the existence of God a 'hard' fact?" Philosophical Review 76: 492-503.

Belnap, Nuel and Mitchell Green. 1994. "Indeterminism and the thin red line." Philosophical Perspectives 8: 365-388.

Cobreros, Pablo and Luca Tranchini. 2014. "Supervaluationism: Truth, value and degree functionality.” Thought 3: 136-144.

De Florio, Ciro and Aldo Frigerio. 2015. "In defense of the timeless solution to the problem of human free will and divine foreknowledge." International Journal for Philosophy of Religion 78: 5-28.

Fine, Kit. 1975. "Vagueness, truth and logic." Synthese 30 (3): 265-300.

Hasker, William. 2001. "The foreknowledge conundrum.” In Issues in Contemporary Philosophy of Religion, edited by Eugene Thomas Long, 97-114. Dordrecht-Boston: Kluwer Academic Publishers.

Keefe, Rosanna. 2000. Theories of Vagueness. Cambridge: Cambridge University Press.

Kretzmann, Norman and Eleonore Stump. 1981. “Eternity.” The Journal of Philosophy 78: 429-458.

Leftow, Brian. 1991. Time and Eternity. Ithaca, NY: Cornell University Press.

Loss, Roberto. 2012. "Branching time, actuality and the puzzle of retrospective determinacy." Thought 1: 16-25. 
MacFarlane, John. 2008. "Truth in the garden of forking paths.” In Relative Truth, edited by Max Kölbel and Manuel García-Carpintero, 81-102. Oxford: Oxford University Press.

Perloff, Michael and Nuel Belnap. 2011. "Future contingents and the battle tomorrow." The Review of Metaphysics 64: 581-602.

Pike, Nelson. 1965. "Divine omniscience and voluntary action.” Philosophical Review 74: 27-46.

Pike, Nelson. 1970. God and Timelessness. New York: Schocken Books.

Prior, Arthur. 1967. Past, Present and Future. Oxford: Claredon Press.

Saunders, John Turk. 1966. “Of God and freedom.” The Philosophical Review 75: 219-225.

Thomason, Richmond. 1970. “Indeterminist time and truth-value gaps.” Theoria 36: 264-281.

van Fraassen, Bas C. 1966. “Singular terms, truth-value gaps and free logic.” Journal of Philosophy 63: 481-495.

Zagzebski, Linda. 2016. “Foreknowledge and free will.” In The Stanford Encyclopedia of Philosophy, edited by Edward N. Zalta. http://plato.stanford.edu/archives/ spr2016/entries/free-will-foreknowledge/ 